\title{
EL PROGRAMA DE CURSOS LIBRES DE LA DIRECCIÓN DE EXTENSIÓN UNIVERSITARIA DE LA UNIVERSIDAD ESTATAL A DISTANCIA (UNED)
}

\author{
Grace Venegas Bonilla \\ Encargada del Programa de Cursos Libres de la Dirección de Extensión Universitaria de la UNED. \\ Ronald Soto Calderón \\ Docente de la Universidad de Costa Rica.
}

Recibido 9-VI-2004 • Aceptado 06-VII-2004

\begin{abstract}
Resumen: Este artículo presenta un recorrido teórico por los fundamentos de la Extensión Universitaria de la UNED, a partir de un análisis de los planteamientos sociofilosóficos que permiten argumentar la implementación del Programa de Cursos Libres, y la revisión de las propuestas de Villarini (1996) y Tünnermann (1999) para una Educación Superior, y de esta manera, articular posteriormente las acciones universitarias como respuestas a una realidad histórico, social y cultural de la sociedad costarricense. A partir de la información que se sistematiza en este articulo, se pretende generar una propuesta de fundamentos teóricos y filosóficos que permitan reflexionar sobre el quehacer universitario con miras a ofrecer una respuesta pertinente a las demandas de diferentes grupos, desde una perspectiva de educación no formal, en este caso desde el Programa de Cursos Libres de la Dirección de Extensión Universitaria de la Universidad Estatal a Distancia, a desarrollar en un artículo posterior.
\end{abstract}

Palabras clave: Cursos libres, educación no formal, extensión universitaria, educación permanente, educación comunitaria, promoción comunitaria.

\section{Introducción}

Este primer acercamiento es un recorrido teórico preliminar a la propuesta de los fundamentos sociofilosóficos del Programa de Cursos Libres de la UNED. La propuesta final será compartida en forma escrita posteriormente.

Esta investigación tiene como intención realizar un estudio sobre las teorías socio-filosóficas que fundamenten la propuesta de Cursos Libres de la Dirección de Extensión de la Universidad Estatal a Distancia (UNED).

El programa de Cursos Libres inició sus funciones en 1992, como producto de un análisis de las necesidades e intereses tanto de la UNED como de diferentes grupos, de poder por un lado proyectarse a la comunidad y por el otro, de acceder a una forma de aprendizaje de tipo no formal. Esto hace eco a lo establecido en la Misión de la Extensión de la UNED, en este sentido, según Consejo Nacional de Rectores (2003).

La Extensión en la Universidad Estatal a Distancia es el quehacer académico que establece un ligamen directo entre la Universidad y el Entorno, y entre aquella y las realidades sociales concretas. 


\begin{abstract}
This article offers a theoretical summary of the fundamentals of UNED's extension programs based on an analysis of thse socio-philosophical ideas that allow us to argue for an implementation of the Free Course Program, and to revise Villarini (1996) and Tunnermann's (1999) proposals for higher education in order then to articulate university actions which respond to the cultural, social and historical reality of Costa Rican society. Based on the information systematized in this article, the study seeks to propose theoretical and philosophical principles that will allow us to reflect on university issues in order to offer a pertinent response to the demands by different groups from a non-formal education perspective, in this case, from the Free Course Program of UNED's Extension Division. This aspect will be developed in a future article.
\end{abstract}

Key words: Non-formal education, university extension (divisions), permanent education, community education, community promotion.
Está dirigido a los sectores de la sociedad que así lo requieran, prioritariamente aquellos con menores posibilidades de acceso a la educación, la cultura y la recreación, ofreciendo un espacio formativo que tenga como meta el mejoramiento de la calidad de vida de los participantes, con el fin de colaborar en el desarrollo de una sociedad más solidaria, participativa, civilista, sensible y con conciencia de su entorno (p. 100).

La oferta de cursos de la UNED, se publicó en 1992, mediante cursos de corta duración. En un inicio el programa funcionó de forma poco constante y sistemática, caracterizándose porque la oferta en algunos años se ofreció y en otros no, es hasta el año 2001, cuando se empieza a proponer una oferta permanente, la cual inicia trabajando mediante el ofrecimiento de 25 cursos libres, de ocho horas cada uno; estos cursos fueron asumidos como un aporte de profesionales de la Institución.

Paulatinamente, estas acciones se transforman y se ofrecen cursos en diversas temáticas, entre ellas material didáctico, yoga, cursos básicos de computación, entre otros de mayor duración, y se incorpora el área de las manualidades.

El programa de Cursos Libres ha sido objeto de una serie de transformaciones, y ha funcionado por tres años consecutivos de manera ininterrumpida, dando respuesta a una matrícula de 300 personas por cuatrimestre aproximadamente. Sin embargo, hasta el momento, el programa no cuenta con ninguna sistematización de la información sobre los cursos y experiencias del programa que le permitan consolidar sus acciones. Es por esta razón, que se pretende mediante esta investigación aportar los conocimientos que argumenten la naturaleza social del Programa de Cursos Libres y especialmente determinar la pertinencia de sus actividades tanto en el contexto educativo de la Dirección de Extensión Universitaria, en particular de la UNED, como en una respuesta a las inquietudes y necesidades de los usuarios.

Uno de los aspectos claves en la oferta de cursos, es la necesidad de analizar la oferta y brindar las mejores respuestas a 
quienes participan de ellos, no solo teniendo en cuenta las demandas de los usuarios, sino también la Misión de la Extensión, es por esta razón que la encargada de este programa ha evidenciado la necesidad de fundamentar las acciones y pertinencia de los cursos libres, tanto en relación sociedad universidad como las implicaciones de la extensión docente, como un pilar del quehacer de la UNED (UNED, 1983). En este sentido, según el acuerdo del Consejo Universitario 2002-357 Las acciones que se brindan por medio de la extensión, deben promover un equilibrio de esfuerzos y recursos, entre los programas o proyectos que persiguen fines de naturaleza social, y aquellos orientados a la generación de ingresos" (Consejo Nacional de Rectores, 2003, p. 101).

La realización de este trabajo es significativa, en tanto aportará las bases teórico-epistemológicas a un programa que requiere de una fundamentación, para consolidar su pertinencia dentro de la Institución y mantener la respuesta social.

Las universidades como instituciones públicas han sido llamadas a ofrecer excelencia académica en el desarrollo de sus acciones educativas (Tünnermann 1980), es por ello, que la presente investigación contribuirá a fortalecer y garantizar la calidad del programa de Cursos Libres de la Dirección de Extensión Universitaria a la cual le ha sido encomendada la tarea de ofrecer capacitación y proponer programas que mejoren la calidad de vida de los ciudadanos, como se destaca en el artículo $45 \mathrm{y}$ 46 del Estatuto Orgánico:

Los programas universitarios de extensión tendrán como propósito principal contribuir a la capacitación del personal al servicio de instituciones públicas y privadas, y servir de instancia permanente para quienes deseen continuar su educación. Para lograr el interés de las comunidades en los asuntos que mejoren la calidad de sus vidas, la UNED organizará actividades tendientes a cumplir esta finalidad. Es así como el énfasis de los programas ha tendido al mejoramiento profesional, dando mayor importancia a los aspectos sociales y a la solución de problemas comunales específicos (UNED, 1983, p. 17).
Con respecto a las cita anterior, la Universidad en nuestro contexto moderno debe de cuestionar constantemente su quehacer con la perspectiva de ofrecer opciones a una sociedad cambiante, en la que las demandas económicas internacionales sumergen al país en cambios de ajuste estructural que afectan todos los ámbitos, entiéndanse estos como social, económico, político y cultural, siendo que es en el ciudadano sobre quien recae el peso de estas presiones.

Por lo expuesto, el Programa de Cursos de Libres nace con la intención de constituirse en una alternativa de estudios para muchas personas que por diversas razones no han tenido acceso al sistema universitario, es preciso incorporar nuevas poblaciones y ofrecer alternativas para distintas etapas del desarrollo del ser humano, proponiendo espacios al alcance de todos y posibilitando el acceso a los conocimientos que requieran tanto para el trabajo como para su adaptación al devenir de la sociedad y al desarrollo de su propia vida.

El programa de Cursos Libres de la UNED, se caracteriza por presentar una amplia oferta de cursos de gran diversidad temática, que se renueva cada cuatrimestre con alternativas de horarios y bajo costo. De esta forma, las personas pueden matricular los cursos porque les interesa para su formación profesional o personal, o escoger otra alternativa de cursos que fomentan la salud mental, la recreación, el esparcimiento y crear mediante ellos, proyectos creativos y artísticos que los introducen en el conocimiento y desarrollo de sus potencialidades, las cuales muchas veces son por ellos desconocidas.

Dentro de las iniciativas de este Programa se ha propuesto la apertura de espacios poco tradicionales en el ambiente universitario, este es el caso del área de manualidades, la cual esta dirigida a una población en su mayoría femenina. Es importante recalcar, que las manualidades no han sido apreciadas dentro de una 
propuesta universitaria, lo cual ha dejado al margen a una gran población que tiene interés en este área.

La UNED, mediante los cursos libres, propicia el ingreso de estas poblaciones al área de las manualidades, para que ellas puedan realizar actividades $\mathrm{y}$ proyectos que fomentan el desarrollo de destrezas y habilidades como los son cursos de: Técnica Quilting, Técnica Country, Muñecas de Lujo en Tela, Arreglos Florales entre otros, siendo esta una respuesta de la Universidad a la comunidad, cumpliendo así con lo que se le ha encomendado desde el Estatuto Orgánico a la UNED, por medio de la Extensión Universitaria, para lo cual ha sido creada la Dirección de Extensión Universitaria.

El aporte de esta investigación contribuirá a conocer a profundidad la naturaleza del programa con perspectivas de mejorar e incorporar innovaciones educativas, tanto en lo pertinente a la capacitación como a los aspectos evaluativos, que permitan garantizar la calidad de los cursos que se imparten desde el Programa.

Por lo mencionado hasta el momento se considera que esta investigación contribuirá a obtener mayor información del quehacer universitario, y a su vez, se constituirá en un trabajo significativo para el desarrollo de los conocimientos en el área curricular.

\section{Visión de hombre desde la UNED}

El aumento poblacional de los años setenta produjo un incremento en la demanda de educación superior por parte de jóvenes, lo que hizo que las tres universidades ya existentes Universidad de Costa Rica (UCR), Instituto Tecnológico de Costa Rica (TEC) y Universidad Nacional (UNA), vieran excedida su capacidad de atención, y por ende, una necesidad insatisfecha de atención a los estudiantes. Por otra parte, la ubicación urbana de las universidades y los requisitos de ingreso dejaban sin oportunidades de estudios superiores a grandes sectores de la población. Esto puso en evidencia que mediante el sistema presencial, era imposible democratizar la educación superior, por lo tanto, era preciso crear una instancia universitaria, que pudiera dar una respuesta a esta población.

La Universidad Estatal a Distancia (UNED) inicia sus labores en el año 1977, nace como una respuesta al aumento en la demanda de educación superior en el país, y en especial, para atender las necesidades de nuevos sectores de la población urgidos de acceder a los bienes educativos que ofrece la educación superior. Esta función se destaca en su Ley de creación, Artículo 1: "Créase la Universidad Estatal a Distancia (UNED), como una institución de educación superior especializada en la enseñanza a través de los medios de comunicación social" (UNED, 1978, p. 1).

Es mediante la metodología a distancia que la UNED ha abierto espacios participativos para poblaciones que por diversas razones, en especial geográficas, económicas y laborales, entre otras, veían obstaculizadas sus aspiraciones de ingreso a la Universidad.

Es evidente que la UNED representa la democratización de la educación superior universitaria, mediante la utilización de todo tipo de medios entre ellos: escritos, audiovisuales y en la actualidad videoconferencia y microcampus.

De las ideas anteriores, se puede extraer que la visión de hombre que posee la Institución, está contenida en la Moción 006 del II Congreso Universitario de la UNED, y en la cual se puede leer

Incorporar en el quehacer universitario un nuevo humanismo entendido como el conocimiento de la realidad concreta del ser humano y el compromiso con la búsqueda de una nueva concepción de este, y de su papel dentro del cosmos. Este nuevo humanismo es el fundamento para el desarrollo de las funciones sustantivas de la UNED" (UNED, 2001, p. 260).

Partiendo entonces de las ideas anteriores, la Institución abre oportunidades 
de estudio desde una visión humanista, fomentando una orientación curricular en la que se destaque una oferta de calidad, la cual se convierta en un instrumento de desarrollo humano, personal y social; respondiendo a las necesidades de la persona y de la sociedad en que se desenvuelve.

No obstante, veinticinco años después de fundada, el panorama social y cultural ha sufrido cambios significativos en todos los ámbitos humanos de nuestra sociedad. La influencia que ejercen los cambios en todos los niveles, entre ellos, las tendencias globalizadoras, el progreso de las ciencias, las nuevas formas de producción acelerada, las incertidumbres de los esquemas valorativos, la omnipresencia de las tecnologías informáticas y telemáticas con sus promesas y amenazas para la educación y desde luego la difusión del conocimiento, proponen a la Universidad, en tanto producto social, como un centro educativo que se convierte en punta de lanza para llevar la vanguardia en la incorporación de estas nuevas tendencias.

Los cambios vertiginosos en el entorno cultural, en las estructuras productivas, en las formas de vinculación social y especialmente la difusión del conocimiento, proponen a las universidades como centros preponderantes para la apropiación de los saberes, y de la conciencia crítica frente a la realidad nacional.

El contexto universitario costarricense, ha sufrido un cambio esencial, el cual consiste en el incremento de la oferta de centros de educación superior, principalmente privados, los que se caracterizan por que sus acciones académicas van amparadas a un claro interés comercial dejando de lado las actividades de acción social.

Ante el panorama anterior, el peso de las demandas sociales recaen en los centros de educación superior público, las cuales por su constitución y filosofía son los entes encargados de la educación permanente y de proponer actividades que tengan como objetivo el desarrollo y mejoramiento de la calidad vida de las personas. Por otra parte las universidades públicas, en la actualidad cuentan con numerosas sedes regionales, que se extienden en buena parte de la geografía nacional, para poder de alguna manera, solventar las demandas de formación que requiere la sociedad.

Al comprender que una de las características fundamentales de los seres humanos es su necesidad de aprender, actividad que se realiza durante toda la vida, hace que se presenten exigencias para encontrar críticamente información válida y ser capaz de convertirla en conocimiento útil para el propio contexto cultural. Como lo señala Delors (1999), son cuatro las razones por las que las universidades se deben de fortalecer; a saber

a) Como centros de conocimiento puro y aplicado y como lugar por excelencia para preparar a los profesores de todos los niveles; b) como proveedoras de personal calificado del más alto nivel; c) como lugar ideal para llevar a cabo la Educación Permanente accesible a los adultos y d) como el vehículo por excelencia para que cada Nación pueda actuar como interlocutora con las comunidades académicas y científicas internacionales (Tünnermann, 1999, p. 4).

Partiendo de la cita anterior la Dirección de Extensión Universitaria siendo consecuente con la visión de humanista asumida por la Institución, potencia el acercamiento entre la comunidad y la universidad, lo que se concreta en acciones caracterizadas con metodologías flexibles, de fácil acceso y sobre todo, que contribuyen a mejorar la calidad de vida de todas las personas.

\section{Dirección de Extensión Universitaria}

Es a la Dirección de Extensión Universitaria a la cual se le ha encomendado la complementariedad de la educación formal por medio de los programas de capacitación, la educación permanente, la actualización y perfeccionamiento y la divulgación a diferentes sectores de la población costarricense. Esto se manifiesta 
en lo propuesto por el Consejo Universitario en 1990, cuando se creó la Dirección de Extensión

(...) aquella dirigida a la comunidad nacional, a las instituciones públicas y a la empresa privada en procura de la educación permanente de los ciudadanos en sus diferentes etapas de su ciclo vital con el objeto de promover tanto el desarrollo de sus potencialidades como la obtención de aquellos conocimientos y técnicas necesarias para que sean más productivos y contribuyan más a su bienestar, al de la familia y al de la sociedad (UNED, 1990, p. 5).

El quehacer de la Dirección de Extensión se determina mediante tres premisas generales que rigen y sirven de guía para las acciones que realiza, las cuales se explicitan comprendiendo la diversidad de segmentos de la población que se deben alcanzar, lo que se destaca en lo dictado por el Estatuto Orgánico la universidad como agente de educación permanente; La universidad como difusora de la cultura y la universidad como agente de promoción comunitaria" (CONARE, 2003 p. 176).

\section{La universidad como agente de la educación permanente}

Se comprende que el devenir histórico de un pueblo se transforma constantemente, y esta condición dialéctica no solamente produce cambios generales, sino también particulares, esto quiere decir que los pueblos se regeneran a partir de la transformación de sus habitantes.

La postmodernidad ha sumido a las personas en la "era de la información", y este concepto amplía y transforma la visión de mundo de los individuos, especialmente por el uso de los medios tecnológicos y la incansable necesidad de conocimiento que posee el ser humano. Esto propone un panorama en el cual la educación no se concluye nunca, la necesidad de saber hace que los sujetos desde que nacen hasta que mueren no se detengan nunca en esta vital actividad. El reto que enfrenta la Universidad es ofrecer alternativas educativas mediante los descubrimientos científicos, tecnológicos, humanísticos y didácticos dado que la sociedad le demanda abrirse a nuevas poblaciones y a nuevas etapas de la vida humana; con el objeto de poner al alcance de los ciudadanos los conocimientos que requieren tanto para sus actividades laborales, como para su adaptación al devenir de la sociedad y al desarrollo de conocimientos diversos, entre ellos las actualizaciones fundamentales para el mejoramiento de la calidad de vida de las personas.

Lo anterior se consolida en la moción 030 del II Congreso Universitario en la que se caracteriza a la Extensión como:

(...) uno de los pilares fundamentales del quehacer académico universitario; por lo tanto, debe ser conceptualizada sobre la base de los siguientes principios: comunicación bidireccional universidad-comunidad, proceso educativo, organizado y dinámico, metodologías flexibles y abiertas, conocimiento estratégico, integración de la universidad con la comunidad, autoeducación, interdisciplinaridad, presencialidad y permanencia, reflexión crítica y autocrítica, creatividad y evaluación permanente (CONARE, 2003, p. 99).

Se requiere por tanto visualizar el proceso educativo, como integrado, único y dinámico, el cual se concrete apelando a diversas estrategias metodológicas y a diversos medios, e instancias que la sociedad ofrece y que pueden convertirse en contextos de aprendizaje. Por lo tanto, las acciones educativas fomentan la integración con procesos socioculturales, económicos, políticos y productivos, según las características de los contextos sociales y regionales, y las condiciones de los participantes.

Es preciso comprender que las prácticas pedagógicas se adecuen a las necesidades y posibilidades de las comunidades, a los valores sociales y a las motivaciones de los participantes y en especial, acentuando la atención en la forma en que ellos conocen, aprenden y piensan para lograr que su aprendizaje sea significativo. Esto conlleva utilizar innovaciones metodológicas que apoyen el proceso de autoaprendizaje, así como la incursión rigurosa de la universidad 
en los diferentes campos del conocimiento a nivel técnico, y en todo aquello que tienda a proponerla como agente de educación permanente de los ciudadanos.

\section{La Universidad como difusora de la cultura}

La identidad cultural se manifiesta de distintas formas pero repercute en todos los ámbitos del quehacer social. El reforzar los rasgos culturales que definen e identifican a una sociedad es el escenario para el desarrollo adecuado a las necesidades de la misma y constituye la base del equilibrio social y rescate de la identidad de los pueblos. Lo cual se destaca en el artículo IX del Acuerdo de Creación de la Dirección de Extensión, en el objetivo f. "Promover la conservación y el desarrollo de la cultura costarricense, mediante la publicación de obras históricas, científicas, artísticas y filosóficas que den a conocer los logros del pasado y los aportes del presente" (CONARE 2003, p. 95).

En particular la sociedad costarricense es compleja, por lo que amerita un reforzamiento de las acciones orientadas al impulso y desarrollo de actividades culturales costarricenses.

Para la UNED como parte del sistema de universidades públicas, y de acuerdo con las poblaciones meta que se le han encomendado, es indispensable implementar acciones desde el campo editorial, el campo audiovisual y el campo del arte y la cultura popular, como sus áreas y dimensiones principales para la difusión de la cultura.

\section{La Universidad como agente de promoción comunitaria}

Los programas de Extensión de la UNED se proponen atender a todos los sectores de la población que así lo requieran, pero dando especial énfasis a aquellos sectores menos favorecidos. Estos proyectos se desarrollan sobre la base de diagnósticos de las necesidades de las comunidades. Estos proyectos están enmarcados dentro de las acciones de promoción y desarrollo de las comunidades promoviendo la interdisciplinaridad.

\section{Estructura de la dirección de extensión en la UNED}

La Dirección de Extensión Universitaria está organizada por medio de programas, dentro de los cuales se desarrollan proyectos que responden al logro de los objetivos de interés institucional. Estos programas de extensión nacen para atender las necesidades de la sociedad costarricense, por lo que no son de carácter permanente, y más bien, son actividades educativas estructuradas que muestran flexibilidad y agilidad para responder oportuna y adecuadamente a las demandas de la comunidad.

Las demandas son atendidas mediante curso libres los cuales deberán ser evaluados por el Centro de Investigación y Evaluación Institucional (CIEI), quien determinará la pertinencia de los programas de Extensión, al menos cada dos años. En caso de que se requieran nuevos programas de Extensión, su aprobación estará a cargo del Consejo Universitario. Los programas aprobados que se encuentran en vigencia a esta fecha según CONARE (2003), son los siguientes:

\section{Programa de desarrollo educativo}

Este programa coordina y desarrolla proyectos dirigidos, preferiblemente, a la actualización y la capacitación del personal docente y administrativo del sistema educativo nacional, así como de aquellos sectores profesionales o no, que requieran desarrollar habilidades y competencias para el mejoramiento continuo. 


\section{Programa de gestión local}

Coordina y desarrolla proyectos que contribuyan al mejoramiento de la calidad de vida de los habitantes, mediante la promoción de actividades tendentes a la participación ciudadana en los procesos de toma de decisiones, facilitando la apropiación del conocimiento para la gestión local, la autogestión y la elaboración de proyectos, la coordinación interinstitucional, la gestión de planes de desarrollo urbano y rural, y el ejercicio de los derechos políticos y culturales.

\section{Programa de promoción cultural y recreativa}

Coordina y desarrolla proyectos de recreación, deporte, promoción del arte, las identidades culturales, en fin de todo aquello que vincule el desarrollo de la cultura nacional, regional y local.

\section{Programa de desarrollo gerencial}

Desarrolla y ejecuta proyectos dirigidos al apoyo, asesoramiento, capacitación y actualización de las empresas públicas y privadas que requieran mejorar su desempeño, tanto en la zona urbana como en la rural.

\section{Programa de idiomas}

Coordina y desarrolla proyectos que favorezcan el aprendizaje de diversas lenguas.

El programa de Desarrollo Gerencial y de Idiomas son programas autofinanciables, esto quiere decir que están llamados a generar ingresos. El Área de Desarrollo Educativo, el Programa de Gestión Local y el Programa de Promoción Cultural y Recreativa no tienen ese mandato.

\section{En el camino de los cursos libres}

El Programa de Cursos Libres opera bajo la modalidad de educación no formal, con cursos de participación y aprovechamiento; estos últimos se caracterizan por tener una duración igual o superior a 30 horas, y tienen reconocimiento ante el Servicio Civil Docente y son considerados para ascender en carrera profesional.

La matrícula se realiza en el período estipulado por la Universidad y la oferta se renueva constantemente. Con esto se incorporan nuevos cursos y temáticas cada cuatrimestre, lo que permite en algunos casos, dar seguimiento a cursos que requieren dar continuidad para incorporar nueva y mayor información, con lo cual se brinda continuidad al nivel superior.

El programa de Cursos Libres se caracteriza por ofrecer cursos con flexibilidad de horario y especialmente a un costo accesible. La mayoría de ellos son de 40 horas (aproximadamente un cuatrimestre) y por su costo en general es de $\phi 15.500 .00$ colones, dinero con el que se cubre el material por trabajar en el curso, exceptuando los cursos del área de manualidades.

La población matriculada en los cursos cada cuatrimestre oscila entre los trescientos y los cuatrocientos estudiantes.

Estos cursos se ofrecen tanto en área de San José, como en todos los lugares del país, donde se cuente con una organización mínima para formar grupos interesados en realizar algún curso en oferta o bien soliciten algún curso específico. Un ejemplo de ello es que, en la actualidad se ofrecen cursos de computación todo el año en los Centros Universitarios de Turrialba y Cañas, y en la Comunidad de San Rafael de Dota se está ofreciendo un curso de Técnica Country a 30 mujeres jefas de hogar, quienes por medio de la Sra. Lilliam Arroyo, Directora de la Escuela Abel Ureña solicitaron la donación del curso, con el objetivo de aprender a trabajar la pintura en madera con fines microempresariales. 
Ha sido un largo camino de esfuerzo significativo para proponer el Programa de Cursos Libres como una actividad universitaria dentro de la Dirección de Extensión y especialmente, demostrar a lo interno de la UNED la pertinencia del programa; pertinencia que la determina la demanda y aceptación por parte de los estudiantes y las comunidades.

\section{Análisis teórico curricular para un programa de cursos libres}

\section{Introducción}

Al reflexionar sobre el trabajo realizado hasta el momento y la realidad de las acciones del Programa de Cursos Libres de la Dirección de Extensión de la UNED, es necesario analizar a la luz de teorías curriculares y académicas estas actividades, de forma que sobre la idea de proponer un sustento crítico producto de un trabajo investigativo sea posible explicar y construir el sentido que tiene este Programa dentro de la Universidad, y a su vez, comprender la naturaleza de las tendencias curriculares a las que se adscribe. Por esta razón, a continuación se efectuará un recorrido teórico por la Propuesta curricular humanista de Villarini, la cual es concomitante con la visión de hombre que tiene la UNED y un estudio de los trabajos de Tünnermann con respecto a la Universidad como institución de educación superior.

\section{Una propuesta curricular humanista: La visión de Villarini (1996)}

Una de las premisas fundamentales de las que parte el humanismo, es comprender al ser humano como único e irrepetible, lo que evidentemente en el terreno educativo lo convierte en el centro de este proceso, es por ello que la conceptualización de un individuo como una unidad biopsicosocial, permite visualizar al estudiante en todas sus dimensiones social, emocional, intelectual y psicológica.

La propuesta curricular de corte humanista, se constituye a partir de los elementos que son pertinentes y significativos para el estudiante, esto implica que su estructura es abierta y flexible, siendo consecuente con el proceso de desarrollo del individuo; esta perspectiva curricular se define como (...) un plan comprensivo y sistemático de las metas educativas más generales del desarrollo humano con el proceso de diseño curricular más específico de carácter académico, deliberativo y técnico" (Villarini, 1996 p. 52).

Desde la visión del autor la función del currículo esta planteada como un instrumento guía para facilitar el aprendizaje y desarrollo en los estudiantes, esto implica considerar que son diferentes los niveles, condiciones ambientales, y psicológicas que son factores que se deben contemplar en una oferta educativa de corte humanista. Es pertinente señalar que uno de los principales aspectos por considerar, es el asunto de los intereses y las experiencias que pueda tener el estudiante, y que como tal, se puede comprender a la educación “(...) como un proceso de continuación y reconstrucción de las experiencias del estudiante" (Villarini, 1996 p. 55).

Lo anterior implica visualizar a un individuo que busca superar sus experiencias dirigiéndolas hacia las necesidades e intereses, que le permiten desarrollarse personal, social y moralmente, lo cual es concomitante con los estudiantes que matriculan un curso libre, y se contemplan dentro de la educación no formal y por ende, quienes toman esta opción lo hacen movidos por variados intereses, y determinados por sus experiencias particulares.

Reflexionar sobre las motivaciones y necesidades que mueven a los seres humanos a aprender o buscar conocimiento son múltiples. La tendencia a la búsqueda de ese conocimiento estará mediatizada por las 
experiencias previas de los individuos, las cuales se transforman en un continuo a lo largo de toda la vida, produciendo que los seres humanos cambien de intereses y apreciaciones conforme van construyendo una representación de la realidad condicionada por aspectos biopsicosociales y culturales.

La cultura es producto de los seres humanos y los seres humanos producto de esta, es el intrincado e inseparable embrague en el que se sustenta el desarrollo y evolución de los seres humanos, dado que esta se construye a partir de que se comparte un código de comunicación común a todos, el lenguaje y que como lo señala Villarini (1996) "El desarrollo humano depende fundamentalmente de las ideas, valores, prácticas, relaciones e instituciones comunitarias y sociales en las que crece la persona" (p. 57).

Teniendo en cuenta los aspectos mencionados, el desarrollo humano puede concebirse como un proceso histórico mediatizado por procesos mentales de (asimilación-acomodación) y evolutivo, en el cual la persona transforma su medio, pero ella es también transformada por este. Esta transformación produce condiciones donde el ser humano cambia constantemente sus formas de ver el mundo, lo que afecta sus intereses, valores y capacidades.

Es evidente que el contexto en el que se desarrolla el ser humano está mediatizado por la cultura, el grupo y la comunidad a la que pertenece. Es en la cultura donde cada individuo se apropia de la experiencia social acumulada y culturalmente compartida por todos los individuos. Un ejemplo de ello es la capacidad (cognitivo-afectiva) para procesar información y construir conocimiento, que le permitan solucionar problemas y tomar decisiones, lo cual realiza la persona, a partir de la apropiación del bagaje cultural que ha estructurado.

Las capacidades y ritmos siempre "particulares" en cada ser humano se manifiestan en sus necesidades, especialmente en sus intereses de aprendizaje, así como en sus particulares formas de inteligencia y estilos de aprendizaje.
Aprender es parte de los procesos adaptativos del ser humano, involucra simultáneamente todas las dimensiones y capacidades del ser: lo cognoscitivo, lo afectivo y lo psicomotor. Por lo que las alternativas educativas no se pueden adscribir únicamente al ámbito académico sino debemos ampliar los horizontes educativos para aquellas personas que desean desarrollar otras habilidades y destrezas mediante cursos particulares.

Desde la perspectiva que se viene trabajando, el aprendizaje se realiza en forma cooperativa, en tanto se aprende con y por medio de otros. Esto permite a la vez, la apropiación de la experiencia histórica, social acumulada y organizada en la cultura.

Cuando los seres humanos aprenden transforman su relación con el mundo, amplían sus espacios en todos los ámbitos y construyen el conocimiento fundamental para solucionar problemas y tomar decisiones. En este sentido, proceso de aprendizaje es personal, y va a depender de las necesidades, intereses y formas de inteligencia entre otros.

\section{Una propuesta de la universidad desde la perspectiva de la Declaración Mundial sobre la Educación Superior (Tünnermann, 1999)}

Las universidades son los centros a los que se les ha encomendado la educación superior, y evidentemente son una construcción social que ha forjado el desarrollo de las sociedades postmodernas.

Son muchos los logros propiciados por la Universidad en los umbrales del siglo XXI, lo cual hizo pertinente analizar el papel de esta Institución con respecto a las tendencias globalizantes y frente a los avances tecnológicos y la velocidad con que la información está disponible, lo cual es producto de los hallazgos científicos y tecnológicos que ha producido la Universidad directa o indirectamente. 
A partir de las tendencias dictaminadas por la UNESCO (1999), tres son los aspectos claves que determinan la posición estratégica de la Educación Superior, entre ellos:

La pertinencia de la Educación Superior se expresa tal vez mejor a través de la variedad de "servicios académicos" que presta a la sociedad”. Más, no basta que la Educación Superior sea más pertinente. Debe también ser de mejor calidad. Pertinencia y calidad deben marchar de la mano, pues son como las dos caras de una misma moneda. El concepto de calidad en la Educación Superior es, a su vez, un concepto multidimencional. No solo abarca las tres funciones clásicas del tríptico misional de que nos habla Ortega y Gasset: docencia, investigación y extensión, lo que se traduce en calidad de su personal docente, calidad de su programa y calidad de sus métodos de enseñanza-aprendizaje, sino que comprende también la calidad de sus estudiantes, de su infraestructura y de su entorno académico.

Todos estos aspectos relacionados con la calidad, más una buena dirección un buen gobierno y una buena gestión administrativa, determinan el funcionamiento de la Universidad y la "imagen institucional" que proyecta a la sociedad en general (Tünnermann, 1999 p. 3).

En la Conferencia Mundial sobre la Educación Superior en 1999 (UNESCO, 1999) se logró proponer a la educación superior en un lugar prioritario en las agendas y debate nacional e internacional para destacar la contribución de la educación superior al desarrollo económico y social en aspectos tales como "(...) la construcción de una cultura de paz, basada en un desarrollo con equidad, justicia, respecto a los derechos humanos, solidaridad y democracia, todo lo cual demanda instituciones de educación superior dotadas de autonomía responsable y libertad académica" (Tünnermann, 1999, p. 4).

El trabajo realizado en esta reunión mundial de países, dejó en claro el aporte fundamental que significan las universidades, especialmente las públicas, su papel en la promoción de los principios democráticos y pensamiento crítico; su aporte a la modernización política y al desarrollo sostenible, a la movilidad social y a la difusión y enriquecimiento de la cultura e identidad nacional.

Cabe destacar que el desarrollo en la ciencia y la tecnología, se ha generado en las universidades públicas, esta es una de las principales razones por las que se considera a la educación superior, como un bien social y un instrumento clave para propiciar los procesos de transformación y modernización de la sociedad.

Son muchos los mandatos y demandas que se le presentan a la Universidad a partir de la Conferencia Mundial, entre ellos destacan, las garantías de acceso de la educación superior que devienen de la Declaración Universal de Derechos Humanos, en la que se debe garantizar el acceso a estudios superiores en condiciones de igualdad para todos, y en función de los méritos de cada uno.

A la Universidad se le ha encomendado la educación permanente, la que propone al estudiante como el centro vital de las actividades universitarias, en tanto la educación es un largo proceso que se lleva a cabo durante toda la vida y cuya finalidad es la de propiciar la integración y actualización de las personas a los nuevos conocimientos.

Por otro lado, los nuevos paradigmas productivos basados en el poder del conocimiento sientan su responsabilidad en la Universidad para el manejo adecuado de la información y fomentar espacios para gestar la apropiación y aplicación de los descubrimientos.

Cabe destacar que se propone para el siglo XXI una educación permanente y sin fronteras, esto quiere decir una educación sin barreras de tiempo y espacio y donde la Universidad debe privilegiar las tareas de extensión y difusión como parte importante de su quehacer académico. Esto sin dejar de lado que la formación de profesionales debe estar enfocada a introducir innovaciones que permitan replanteamientos en los graduados y como se menciona en UNESCO (citado por Tünnermann, 1999) 
(...) La educación superior necesita introducir métodos pedagógicos basados en el aprendizaje para formar nuevos graduados que aprendan a aprender a emprender, de suerte que sean capaces de generar sus propios empleos e incluso crear entidades productivas que contribuyan a abatir el flagelo del desempleo (p. 14).

Las instituciones de educación deben fomentar iniciativas no solamente formativas a los graduados y de un conocimiento lineal, sino incentivar el desarrollo de destrezas y habilidades psicomotoras que permitan la generación de conocimiento; de esta forma los alumnos estarían incorporando el bagaje fundamental para: aprender a aprender, aprender a conocer, a convivir y a ser.

Es fundamental destacar que la Universidad posee,

(...) una especie de "autoridad intelectual", que ayuda a la sociedad a reflexionar sobre sus acciones, mediante sus actividades académicas y civilistas. Ayuda a incentivar la crítica prospectiva frente a las realidades de los países, como lo destaca la Declaración Mundial La educación superior debe fortalecer su capacidad de análisis crítico de anticipación y de visión prospectiva; para elaborar propuestas alternativas (Tünnermann, 1999 p. 16).

Desde el escenario universitario es donde la función crítica y civilista permite difundir y defender valores universalmente aceptados y en particular la justicia, la paz, la libertad, la igualdad y solidaridad.

La misión cultural es otro aspecto encomendado a la Universidad como centro de rescate de los valores auténticos e idiosincrásicos de los pueblos. Las movilizaciones de tendencias globalizadoras son amenazas donde la identidad de los pueblos corren el riesgo de entrar en una homogenización cultural, donde se pueden perder rasgos culturales ligados a comunidades locales, regionales y nacionales. Esto compromete a las universidades a un trabajo investigativo sobre los valores culturales y sus escenarios, además, mediante acciones de concienciación preservar la identidad de los pueblos.
Es preciso comprender que la Universidad tiene a cargo aspectos fundamentales para el desarrollo del devenir histórico de los pueblos, en tanto es la llamada a promover actitudes reflexivas y críticas fomentando la libertad como uno de sus principios significativos, oponiéndose a toda forma de opresión; su sensibilidad ante los programas sociales la dispone a emprender programas de proyección social; y sus acciones deben extenderse a todos los sectores. Y en especial en la actualidad, debe participar en los esfuerzos dirigidos a promover cambios estructurales en la sociedad para fomentar la justicia social.

La nueva visión de la educación superior presentada en la Declaración Mundial, se basa en varios ejes a saber:

(...) a) la igualdad de acceso; b) el fortalecimiento de la participación y promoción del acceso de las mujeres; c) la promoción del saber mediante la investigación en los ámbitos de la ciencia, el arte y las humanidades y la difusión de sus resultados; d) la orientación a largo plazo de la pertinencia; e) el reforzamiento de la cooperación con el mundo del trabajo y el análisis y la previsión de las necesidades de la sociedad; f) la diversificación como medio de reforzar la igualdad de oportunidades; g) la introducción de métodos educativos innovadores: pensamiento crítico y creatividad; y h) el personal y los estudiantes, principales protagonistas de la educación superior (Tünnermann, 1999 p. 17).

Son muchas las recomendaciones y demandas claramente formuladas en la Declaración Mundial de la Educación Superior, a la Universidad, esto ratifica la vigencia y permanencia en tanto institución garante de todas las formas de libertad y participación democrática de los pueblos.

A partir de este tránsito por Villarini (1996) y Tünnermann (1999), es que se extraen ideas que permitirán el desarrollo de una propuesta de fundamentos sociofilosóficos para programa de Cursos Libres de la UNED, la que se concretará en un documento que se publicará posteriormente. 


\section{A manera de consideraciones finales}

El Programa de Cursos Libres de la UNED, al estar inserto y ser parte de la Dirección de Extensión Universitaria se debe adscribir a una visión humanista tomando al ser humano en su dimensión biopsicosocial, desde una perspectiva holística e integradora. De esta forma el ser humano es una unidad única e irrepetible, la cual está determinada por un proceso sociohistórico particular y que por ello tiene tendencias, experiencias e intereses marcados por estas condiciones.

Desde esta perspectiva el proceso de aprendizaje en cada persona está determinado de distinta forma, mostrando todos múltiples capacidades intelectuales y dimensiones en cuanto a estilos de aprendizaje, las cuales se manifiestan en las habilidades y destrezas que las personas pueden poseer, muchas de ellas son evidentes por el tipo de actividades en que están inmersas y otras se encuentran aún sin descubrir. Dado que el potencial humano se desarrolla en razón de esfuerzos tanto físicos como mentales a los que se expongan las personas y que como lo señala Villarini “(...) Todo ser humano tiene un potencial de desarrollo, una zona próxima de desarrollo, que está determinado por el desarrollo previo alcanzado y el que se puede inducir a través de la mediatización cultural y la interacción con personas en etapas más altas del desarrollo" (p. 56).

Por otro lado y según el recorrido realizado al ser definida la competencia humana por Villarini como “(...) la habilidad general producto del dominio de conceptos, destrezas y actitudes" (p. 61) para efectos de la perspectiva humanista de la Universidad, se plantea un ser humano con capacidad de decisión, quien demuestra claridad en razón de lo que hace, por qué lo hace, y tiene una concepción sobre su actuación, esto se acompaña de la actitud o disposición para hacer uso del conocimiento, el cual ha sido producto de una construcción particular de la historia de cada persona.

Teniendo en cuenta las ideas esbozadas en el texto y en las anteriores conclusiones se puede decir que la Universidad, por lo tanto, debe de atender las demandas sociales del entorno, particularmente de los sectores más desfavorecidos. Esto obliga a la Universidad a una revisión entre su quehacer y los servicios que la sociedad requiere. En la Declaración Mundial de la Educación Superior se proclama que la educación superior debe velar por las necesidades sociales, en este sentido ella

(...) debe reforzar sus funciones de servicio a la sociedad, y más concretamente sus actividades encaminadas a erradicar la pobreza, la intolerancia, la violencia, el analfabetismo, el hambre, el deterioro del medio ambiente y las enfermedades, principalmente mediante un planeamiento interdisciplinario y transdisciplinario para analizar los problemas y las cuestiones planteadas (Tünnermann, 1999 p. 18).

Otro aspecto a considerar se relaciona con las actividades laborales, es preciso destacar que la universidad debe afinar sus sentidos para lo cual debe analizar el mundo del trabajo, “(...) atendiendo la diversidad y movilidad de las demandas del sector laboral y de la economía, solo puede ser atendida adecuadamente por un sistema debidamente integrado, la educación postsecundaria, que ofrezca una amplia oferta educativa a demandantes de cualquier edad" (Tünnermann, 1999, p.19).

Como última consideración, pero no menos importante de este trabajo, es preciso señalar que la propuesta de la educación permanente encomendada a la universidad conlleva a introducir o incentivar aquellas prácticas educativas que se caractericen por su flexibilidad en sus acciones académicas. Y como respuesta a los requerimientos de la sociedad en todos los aspectos.

El aspecto de la calidad en la educación superior pone en relieve la evaluación de las acciones académicas para el mejoramiento de las actividades, permitiendo la emisión de juicios para la toma de 
decisiones, es a partir de estas reflexiones que se pretende generar una propuesta sociofilosófica para el Programa de Cursos Libres de la UNED, la cual será compartida posteriormente.

\section{Referencias bibliográficas}

Barrantes, R. Educación a Distancia. San José, Costa Rica: EUNED. 1998.

Colás B. Ma. P. y otras. Investigación Educativa. Sevilla, España: Ediciones Alfar. 1994.

Consejo Nacional de Rectores (CONARE). La Extensión y la Acción Social en las Instituciones de Educación Superior Universitaria Estatal de Costa Rica. San José, Costa Rica: CONAREOPES. 2003.

Delors, J. La educación encierra un tesoro. Madrid, España: Ediciones UNESCO. 1996.

Delors, J. Declaración sobre la Educación Superior en América Latina y el Caribe. Plan de Acción En la educación superior en el Siglo XXI. Visión y acción. París: UNESCO. 1999.

Dobles Y., C. y otras. Investigación en educación: Procesos, interacciones, construcciones. San José, Costa Rica: EUNED. 1996.

Gimeno Sacristán, L. El currículo: una reflexión sobre la práctica. Madrid, España: Ediciones Morata. 1996.

Méndez, C. Metodología: Diseño y desarrollo del proceso de investigación. Bogotá, Colombia: MacGraw Hill Interamericana S.A. 2001.

Taylor, S.J. y Bogdan, R. Introducción a los métodos cualitativos de investigación. Barcelona, España: Ediciones Paidós. 1992.
Torres S., J. Globalización e interdisciplinaridad: el currículum integrado. Madrid, España: Ediciones Morata. 1996.

Tünnermann B., C. De la Universidad y su problemática. México:Dirección General de Publicaciones UNAM. 1980.

Universidad Estatal a Distancia. Programa de Cursos Libres: Informe de la Dirección de Extensión: Archivo Central. UNED. 1993.

Universidad Estatal a Distancia. Programa Cursos Libres: Informe de la Dirección de Extensión: Archivo Central. UNED. 1994.

Universidad Estatal a Distancia. Instructivo de Matrícula: Escuela de Estudios Generales. Costa Rica. UNED. 1995.

Universidad Estatal a Distancia. Programa Cursos Libres: Informe de la Dirección de Extensión: Archivo Central. UNED. 1995.

Universidad Estatal a Distancia. Factores Claves para el Éxito. Sabanilla: Vicerrectoría Académica. 2001.

Universidad Estatal a Distancia. Informe de labores del Rector 2000. Sabanilla: EUNED. 2001.

Universidad Estatal a Distancia. Acuerdo Consejo Universitario. Sesión 1585357: Consejo Universitario. 2002.

Universidad Estatal a Distancia. Instructivo de Matrícula. San José, Costa Rica: EUNED. 2003.

Villarini, A. El currículum orientado hacia el desarrollo humano integral. Río Piedras, Puerto Rico: Biblioteca del Pensamiento Crítico. 1996.

Wittrock, M. La investigación de la enseñanza, I Enfoque, teorías y métodos. Barcelona, España: Ediciones Paidós. 1997. 